\title{
Next-to-leading order evolution of transversity distributions and Soffer's inequality
}

\author{
Werner Vogelsang \\ Theory Division, CERN, CH-1211 Geneva 23, Switzerland
}

(Received 1 July 1997; published 19 December 1997)

\begin{abstract}
We present a calculation of the two-loop splitting functions for the evolution of the twist-2 "transversity", parton densities of transversely polarized nucleons. We study the implications of our results for Soffer's inequality for the case of valence quark densities. [S0556-2821(98)01603-8]

PACS number(s): 13.88.+e, 12.38.Bx
\end{abstract}

\section{INTRODUCTION}

The past few years have seen much progress in our knowledge about the nucleon's spin structure thanks to the experimental study of the spin asymmetries $A_{1}^{N}\left(x, Q^{2}\right)(N$ $=p, n, d)$ in deep-inelastic scattering (DIS) with longitudinally polarized lepton beams and nucleon targets. Such experiments yield information on the "longitudinally polarized" (or "'helicity-weighted') parton distributions of the nucleon, which will be denoted as

$$
\Delta_{L} f\left(x, Q^{2}\right) \equiv f^{\rightarrow}\left(x, Q^{2}\right)-f^{\leftarrow}\left(x, Q^{2}\right),
$$

where $f^{\rightarrow}\left(x, Q^{2}\right)\left[f^{\leftarrow}\left(x, Q^{2}\right)\right]$ is the probability at scale $Q$ of finding a parton-type $f[f=q, \bar{q}, g]$ in a longitudinally polarized nucleon (for definiteness, say, a proton) with its spin aligned (antialigned) with the proton spin and carrying the fraction $x$ of the proton's momentum. Taking the sum instead of the difference in Eq. (1) one obtains the usual unpolarized parton distributions of the proton,

$$
f\left(x, Q^{2}\right) \equiv f^{\rightarrow}\left(x, Q^{2}\right)+f^{\leftarrow}\left(x, Q^{2}\right) .
$$

Apart from $f\left(x, Q^{2}\right)$ and $\Delta_{L} f\left(x, Q^{2}\right)$, a third type of parton density can be defined which describes the properties of transversely polarized nucleons [1-4]:

$$
\Delta_{T} f\left(x, Q^{2}\right) \equiv f^{\uparrow}\left(x, Q^{2}\right)-f^{\downarrow}\left(x, Q^{2}\right),
$$

where now $f^{\uparrow}\left(x, Q^{2}\right)\left[f^{\downarrow}\left(x, Q^{2}\right)\right]$ stands for the probability of finding a parton-type $f$ in a transversely polarized proton with its spin parallel [antiparallel] to the proton spin. These parton densities are usually referred to as "transversity" distributions. They complete the twist- 2 sector of nucleonic parton distributions and are therefore as interesting in principle as $f\left(x, Q^{2}\right)$ and $\Delta_{L} f\left(x, Q^{2}\right)$. Unlike the case of the latter two, there is no gluonic transversity distribution at leading twist $[5,6]$.

Unfortunately, nothing is known as yet experimentally about the transversity distributions. As was shown in [2-4], it is not possible to measure them in inclusive deep-inelastic scattering because they are chirally odd. It is widely accepted that Drell-Yan lepton pair production in collisions of transversely polarized protons offers the best possibility to get access to the $\Delta_{T} f[1-4,7-10]$, and the BNL Relativistic Heavy Ion Collider (RHIC) spin physics program comprises experiments of this kind [11].

There being a shortage of experimental information on the transversity distributions, there has been quite some effort on the theoretical side, aiming at the question of whether the $\Delta_{L} f$ and $\Delta_{T} f$ are entirely independent of each other. This is not expected really because one clearly has

$$
f^{\rightarrow}\left(x, Q^{2}\right)+f^{\leftarrow}\left(x, Q^{2}\right)=f^{\uparrow}\left(x, Q^{2}\right)+f^{\downarrow}\left(x, Q^{2}\right) .
$$

In fact, Soffer has derived an interesting nontrivial inequality $[12,7]$,

$$
\left|\Delta_{T} f\right| \leqslant \frac{1}{2}\left(f+\Delta_{L} f\right) \equiv f^{\rightarrow}
$$

which is valid separately for each quark flavor.

Soffer's inequality can be derived in the context of a parton model $[12,7,13]$. The question immediately rises of whether the inequality is maintained when QCD is applied [13-15]. There are two potential sources of concern here. First, as indicated in Eqs. (1)-(3), the parton distributions evolve with $Q^{2}$. Since the corresponding evolution kernels at leading order (LO) [16,2] turn out to be different for the $f\left(x, Q^{2}\right), \Delta_{L} f\left(x, Q^{2}\right), \Delta_{T} f\left(x, Q^{2}\right)$, it is not a priori clear that the inequality is preserved by QCD evolution. It turns out [15], however, that this is the case. The crucial point here is that the difference of the involved flavor nonsinglet LO Altarelli-Parisi evolution kernels is always positive,

$$
\frac{1}{2}\left(P_{q q}^{(0)}(x)+\Delta_{L} P_{q q}^{(0)}(x)\right)-\Delta_{T} P_{q q}^{(0)}(x)=C_{F}(1-x) \geqslant 0,
$$

where $C_{F}=4 / 3$. In Eq. (6) $P_{q q}^{(0)}, \Delta_{L} P_{q q}^{(0)}$ are the unpolarized and longitudinally polarized LO quark-to-quark splitting functions, respectively, which have been calculated in [16] and are equal because of helicity conservation. $\Delta_{T} P_{q q}^{(0)}$ is their transversely polarized counterpart of [2]. From Eq. (6) it follows that if one assumes validity of Soffer's inequality (5) at some input scale $Q_{0}$, the inequality will still be fulfilled at any higher scale, at least in the context of LO evolution. Strictly speaking, this statement only follows for flavor nonsinglet, in particular valence, quark densities, which is due to the lack of gluons in the case of transversity distri- 
butions. However, as was shown in [15], the picture does not change when the full LO singlet evolution is taken into account for the $f\left(x, Q^{2}\right), \Delta_{L} f\left(x, Q^{2}\right)$ on the right-hand side of (5).

Secondly, there is the question of what role higher order QCD corrections play in Soffer's inequality. These enter in two ways, namely via the corrections to the splitting functions governing the evolution of the parton distributions, and as corrections to the cross sections for the processes which are used to extract information on the parton distributions from experiment in order to see whether they satisfy Eq. (5). As is well-known, the split-up of the higher order corrections into corrections to the splitting functions and to the cross sections is not unique but depends on the factorization scheme adopted, which in turn implies that the parton densities themselves are scheme-dependent beyond LO. Clearly, one is free to choose the factorization schemes independently for the $f\left(x, Q^{2}\right), \Delta_{L} f\left(x, Q^{2}\right)$ and $\Delta_{T} f\left(x, Q^{2}\right)$, and it will in principle always be possible to violate Eq. (5) by an arbitrarily large amount by adopting certain "sufficiently incompatible" schemes for the three densities. One therefore has to ask in which factorization scheme(s) it makes sense to try to check Eq. (5) beyond LO. In other words, what should be the meaning of Eq. (5) in higher orders? This question has also been addressed in [14] and will be one key issue of this paper. We will provide all ingredients for the next-to-leading order (NLO) framework of transversity distributions, which will serve to enable a check of Soffer's inequality to NLO.

As was pointed out earlier, the Drell-Yan process with transversely polarized hadrons appears to be the most likely candidate for measurements of the transversity distributions. Much theoretical work has therefore focused on this process [1-4,7-10]. In particular, the NLO corrections to the transversely polarized Drell-Yan have been calculated in [8] and $[9,17]$, within different factorization schemes. The result of the latter calculation $[9,17]$ was used in [14] for a first assessment of the impact of NLO corrections on Soffer's inequality. However, for future NLO analyses of (hopefully forthcoming) experimental data, knowledge of the NLO corrections to the Drell-Yan cross section alone cannot be sufficient as data will necessarily be taken at various different values of the invariant mass of the Drell-Yan muon pair which sets the hard scale for the process. According to the previous paragraph, the NLO corrections to the $Q^{2}$-evolution of the transversity distributions also have to be known in order to perform a complete and consistent NLO calculation. The main result of this paper will therefore be the presentation of the NLO evolution kernels for the transversity distributions - only when these are available can Soffer's inequality be meaningfully studied beyond LO. The method we will use to calculate the NLO transversity splitting functions will be the one used by Curci, Furmanski and Petronzio in the unpolarized case $[18,19]$ (see also [20]) and which was also utilized recently [21] to obtain the NLO evolution kernels for the longitudinally polarized parton distributions $\Delta_{L} f$.

The remainder of this paper is organized as follows. In Sec. II we will outline the framework for our calculations. Also in our case the transversely polarized Drell-Yan process will be the starting point. Section III will give details of the calculation of the NLO transversity splitting functions. Sec- tion IV will present the results and focus on the validity of Soffer's inequality beyond LO. Finally, we will draw our conclusions in Sec. V.

\section{FRAMEWORK}

We begin by defining the spin-dependent cross section for dimuon production by protons with transverse polarization along, say, the $x$-axis:

$$
\frac{\tau d \Delta_{T} \sigma}{d \tau d \phi} \equiv \frac{1}{2}\left(\frac{\tau d \sigma^{\uparrow \uparrow}}{d \tau d \phi}-\frac{\tau d \sigma^{\uparrow \downarrow}}{d \tau d \phi}\right)
$$

where the superscript $\uparrow \uparrow(\uparrow \downarrow)$ denotes parallel (antiparallel) setting of the transverse spins of the incoming protons. Furthermore, $\tau \equiv M^{2} / S$, where $M$ is the invariant mass of the muon pair and $\sqrt{S}$ the center-of-mass energy of the hadronic collision; $\phi$ is the azimuthal angle of one of the muons and is counted relative to the axis defined by the transverse polarizations. For the discussion to follow in this section it is convenient to temporarily go to Mellin moment space where we can perform the $Q^{2}$-evolutions more explicitly. The Mellin moments of the cross section are defined by

$$
\frac{d \Delta_{T} \sigma^{n}}{d \phi} \equiv \int_{0}^{1} d \tau \tau^{n-1} \frac{\tau d \Delta_{T} \sigma}{d \tau d \phi}
$$

Including the NLO corrections to this cross section [as calculated in a certain factorization scheme such as the modified minimal subtraction $(\overline{\mathrm{MS}})$ scheme], one has the generic expression $^{1}[8,9]$

$$
\frac{d \Delta_{T} \sigma^{n}}{d \phi}=\frac{\alpha_{e m}^{2}}{9 S} \cos (2 \phi) \Delta_{T} H^{n}\left(M^{2}\right)\left(1+\frac{\alpha_{s}\left(M^{2}\right)}{2 \pi} \Delta_{T} C_{q}^{\mathrm{DY}, n}\right),
$$

where

$$
\begin{aligned}
\Delta_{T} H^{n}\left(Q^{2}\right) \equiv & \sum_{q} e_{q}^{2}\left[\Delta_{T} q_{1}^{n}\left(Q^{2}\right) \Delta_{T} \bar{q}_{2}^{n}\left(Q^{2}\right)\right. \\
& \left.+\Delta_{T} \bar{q}_{1}^{n}\left(Q^{2}\right) \Delta_{T} q_{2}^{n}\left(Q^{2}\right)\right] .
\end{aligned}
$$

Here we have restored for the moment the possibility of the scattering of two different hadrons labelled " 1 ," " 2 ,", even though anything apart from $p p$ scattering will presumably not be realistic experimentally. We will keep this notation when dealing with the function $\Delta_{T} H$, but otherwise suppress the indices " 1, ," " 2 ," in the following. The Mellin moments of the quark distributions $\Delta_{T} q$ and the $\mathcal{O}\left(\alpha_{s}\right)$ corrections $\Delta_{T} C_{q}^{\mathrm{DY}}$ are defined in analogy with Eq. (8), e.g. $\Delta_{T} q^{n}\left(Q^{2}\right)$ $\equiv \int_{0}^{1} d x x^{n-1} \Delta_{T} q\left(x, Q^{2}\right)$.

When including the $\mathcal{O}\left(\alpha_{s}\right)$ corrections in (9), it is crucial that the parton distributions are evolved to NLO accuracy as

\footnotetext{
${ }^{1}$ Here we only consider photon exchange. The contributions from $Z^{0}$ exchange and $\gamma-Z^{0}$ interference can be straightforwardly included by making use of Eq. (3) of [9].
} 
well. Since there are no gluons involved in the case of the transversity distributions, their evolution equations reduce to simple nonsinglet type equations. Introducing

$$
\Delta_{T} q_{ \pm}^{n} \equiv \Delta_{T} q^{n} \pm \Delta_{T} \bar{q}^{n}
$$

one has

$$
\begin{aligned}
\Delta_{T} H^{n}\left(Q^{2}\right)= & \frac{1}{2} \sum_{q} e_{q}^{2}\left[\Delta_{T} q_{+, 1}^{n}\left(Q^{2}\right) \Delta_{T} q_{+, 2}^{n}\left(Q^{2}\right)\right. \\
& \left.-\Delta_{T} q_{-, 1}^{n}\left(Q^{2}\right) \Delta_{T} q_{-, 2}^{n}\left(Q^{2}\right)\right]
\end{aligned}
$$

and the evolution equations (see, e.g., [20])

$$
\frac{d}{d \ln Q^{2}} \Delta_{T} q_{-}^{n}\left(Q^{2}\right)=\Delta_{T} P_{q q,-}^{n}\left(\alpha_{s}\left(Q^{2}\right)\right) \Delta_{T} q_{-}^{n}\left(Q^{2}\right),
$$

$$
\begin{aligned}
& \frac{d}{d \ln Q^{2}}\left(\Delta_{T} q_{+}^{n}-\Delta_{T} q^{\prime n}\right)\left(Q^{2}\right)=\Delta_{T} P_{q q,+}^{n}\left(\alpha_{s}\left(Q^{2}\right)\right)\left(\Delta_{T} q_{+}^{n}\right. \\
& \left.-\Delta_{T} q^{\prime \prime}{ }_{+}\right)\left(Q^{2}\right), \\
& \frac{d}{d \ln Q^{2}} \Delta_{T} \Sigma^{n}\left(Q^{2}\right)=\Delta_{T} P_{\Sigma \Sigma}^{n}\left(\alpha_{s}\left(Q^{2}\right)\right) \Delta_{T} \Sigma^{n}\left(Q^{2}\right),
\end{aligned}
$$

where $q, q^{\prime}$ are different quark flavors and $\Delta_{T} \Sigma^{n} \equiv \Sigma_{q} \Delta_{T} q_{+}^{n}$. Note that the first moment $(n=1)$ in Eq. (13) corresponds to the evolution of the nucleon's tensor charge [3,22]. The splitting functions $\Delta_{T} P_{q q, \pm}\left(\alpha_{s}\left(Q^{2}\right)\right), \Delta_{T} P_{\Sigma \Sigma}$ are taken to have the following perturbative expansion:

$$
\Delta_{T} P_{i i}^{n}\left(\alpha_{s}\right)=\left(\frac{\alpha_{s}}{2 \pi}\right) \Delta_{T} P_{q q}^{(0), n}+\left(\frac{\alpha_{s}}{2 \pi}\right)^{2} \Delta_{T} P_{i i}^{(1), n}+\cdots,
$$

$\{i i\}=\{q q, \pm\},\{\Sigma \Sigma\}$. As indicated, $\Delta_{T} P_{q q,+}, \Delta_{T} P_{q q,-}$ and $\Delta_{T} P_{\Sigma \Sigma}$ are all equal at LO. It is convenient to write [20]

$$
\begin{aligned}
& \Delta_{T} P_{q q, \pm}^{(1), n} \equiv \Delta_{T} P_{q q}^{(1), n} \pm \Delta_{T} P_{q \bar{q}}^{(1), n}, \\
& \Delta_{T} P_{\Sigma \Sigma}^{(1), n} \equiv \Delta_{T} P_{q q,+}^{(1), n}+\Delta_{T} P_{q q, P S}^{(1), n} .
\end{aligned}
$$

It will actually turn out that $\Delta_{T} P_{q q, P S}^{(1), n} \equiv 0$, so that Eqs. (14), (15) can be replaced by

$$
\frac{d}{d \ln Q^{2}} \Delta_{T} q_{+}^{n}\left(Q^{2}\right)=\Delta_{T} P_{q q,+}^{n}\left(\alpha_{s}\left(Q^{2}\right)\right) \Delta_{T} q_{+}^{n}\left(Q^{2}\right) .
$$

Needless to say that the NLO splitting functions have to be known in the same factorization scheme as the corrections to the cross section, $\Delta_{T} C_{q}^{\mathrm{DY}}$.

The solution to Eqs. (13), (19) is well-known (see, e.g., [23]) and reads

$$
\begin{aligned}
\Delta_{T} q_{ \pm}^{n}\left(Q^{2}\right)= & \left(1+\frac{\alpha_{s}\left(Q_{0}^{2}\right)-\alpha_{s}\left(Q^{2}\right)}{\pi \beta_{0}}\left[\Delta_{T} P_{q q, \pm}^{(1), n}\right.\right. \\
& \left.\left.-\frac{\beta_{1}}{2 \beta_{0}} \Delta_{T} P_{q q}^{(0), n}\right]\right) \\
& \times\left(\frac{\alpha_{s}\left(Q^{2}\right)}{\alpha_{s}\left(Q_{0}^{2}\right)}\right)^{-2 \Delta_{T} P_{q q}^{(0), n} / \beta_{0}} \Delta_{T} q_{ \pm}^{n}\left(Q_{0}^{2}\right)
\end{aligned}
$$

with the input distributions $\Delta_{T} q_{ \pm}^{n}\left(Q_{0}^{2}\right)$ at the input scale $Q_{0}$. Here and in all previous NLO equations one has to use the two-loop expression for the strong coupling,

$$
\frac{\alpha_{s}\left(Q^{2}\right)}{4 \pi} \simeq \frac{1}{\beta_{0} \ln Q^{2} / \Lambda_{\overline{\mathrm{MS}}}^{2}}-\frac{\beta_{1}}{\beta_{0}^{3}} \frac{\ln \ln Q^{2} / \Lambda_{\overline{\mathrm{MS}}}^{2}}{\left(\ln Q^{2} / \Lambda_{\overline{\mathrm{MS}}}^{2}\right)^{2}}
$$

with the QCD scale parameter $\Lambda_{\overline{\mathrm{MS}}}$ and $\beta_{0}=11-2 f / 3, \beta_{1}$ $=102-38 f / 3, f$ being the number of active flavors. We note that all LO expressions are entailed in the above equations by setting the NLO quantities $\Delta_{T} C_{q}^{\mathrm{DY}}, \Delta_{T} P_{\ldots}^{(1)}, \beta_{1}$, to zero.

Formulas exactly analogous to the above hold for the unpolarized and the longitudinally polarized cases. The only complication here is that there are obviously contributions by gluons to the Drell-Yan cross section and to the evolution of the quark singlet combination $\left(\Delta_{L}\right) \Sigma^{n}$. Let us assume for the moment that we are able to eliminate these experimentally by taking suitable nonsinglet combinations of Drell-Yan cross sections for various configurations of scattering hadrons. In particular, when considering the difference of the cross sections for $p \bar{p}$ and $p p$ scattering, the function $\Delta_{T} H^{n}$ reduces to pure valence,

$$
\Delta_{T} H^{n}\left(Q^{2}\right)=\sum_{q} e_{q}^{2} \Delta_{T} q_{-}^{n}\left(Q^{2}\right) \Delta_{T} q_{-}^{n}\left(Q^{2}\right)
$$

where all parton distributions refer to the proton, and with similar expressions for the unpolarized and longitudinally polarized cases. We then only need to consider Eqs. (9), (13) which exactly carry over to the unpolarized case [by omitting all $\Delta_{T}$ and replacing $\cos (2 \phi)$ by 2$]$ or to the longitudinally polarized case [by replacing $\Delta_{T}$ by $\Delta_{L}$ and $\cos (2 \phi)$ by -2 ], respectively.

We now adopt the Drell-Yan process as the process defining the (NLO) parton distributions and follow [14] to argue that (if at all) Soffer's inequality for the valence densities should be valid beyond LO if we choose a factorization scheme in which the nonsinglet Drell-Yan cross sections for the unpolarized, the longitudinally polarized and the transversely polarized cases all individually maintain their respective LO forms. This appears reasonable since the parton distributions are then directly related to a physical (observable) quantity. For example, in case of the cross section for transverse polarization it means

$$
\frac{d \Delta_{T} \sigma^{n}}{d \phi}=\frac{\alpha_{e m}^{2}}{9 S} \cos (2 \phi) \Delta_{T} \widetilde{H}^{n}\left(M^{2}\right)
$$


where $\Delta_{T} \widetilde{H}^{n}\left(Q^{2}\right)$ is defined as in (10),(22) but in terms of the NLO parton densities $\Delta_{T} \widetilde{q}^{n}\left(Q^{2}\right)$ in the new scheme. Inserting (20) into (9), expanding in $\alpha_{s}$ and equating with (23) we find

$$
\begin{aligned}
\Delta_{T} \widetilde{q}_{-}^{n}\left(Q^{2}\right)= & \left(1+\frac{\alpha_{s}\left(Q_{0}^{2}\right)-\alpha_{s}\left(Q^{2}\right)}{\pi \beta_{0}} \Delta_{T} \mathcal{E}_{-}^{n}\right) \\
& \times\left(\frac{\alpha_{s}\left(Q^{2}\right)}{\alpha_{s}\left(Q_{0}^{2}\right)}\right)^{-2 \Delta_{T} P_{q q}^{(0), n} / \beta_{0}} \Delta_{T} \widetilde{q}_{-}^{n}\left(Q_{0}^{2}\right)
\end{aligned}
$$

in the new scheme, where

$$
\Delta_{T} \mathcal{E}_{-}^{n} \equiv \Delta_{T} P_{q q,-}^{(1), n}-\frac{\beta_{0}}{4} \Delta_{T} C_{q}^{\mathrm{DY}, n}-\frac{\beta_{1}}{2 \beta_{0}} \Delta_{T} P_{q q}^{(0), n}
$$

is a combination of ( $\overline{\mathrm{MS}})$ NLO quantities which is manifestly independent of the factorization scheme due to the fact that the Drell-Yan cross section in Eq. (23) is a physical quantity. As a necessary condition for Eq. (5) to hold for the valence densities, its moments (for real $n>0$ ) must also satisfy Soffer's inequality. Thus

$$
\begin{aligned}
\frac{2\left|\Delta_{T} \widetilde{q}_{-}^{n}\left(Q^{2}\right)\right|}{\widetilde{q}_{-}^{n}\left(Q^{2}\right)+\Delta_{L} \widetilde{q}_{-}^{n}\left(Q^{2}\right)}= & \frac{1+\left(\alpha_{s}\left(Q_{0}^{2}\right)-\alpha_{s}\left(Q^{2}\right)\right) \Delta_{T} \mathcal{E}_{-}^{n} /\left(\pi \beta_{0}\right)}{1+\left(\alpha_{s}\left(Q_{0}^{2}\right)-\alpha_{s}\left(Q^{2}\right)\right)\left[\mathcal{E}_{+}^{n}-\frac{2 \widetilde{q}_{-}^{n}\left(Q_{0}^{2}\right)}{\widetilde{q}_{-}^{n}\left(Q_{0}^{2}\right)+\Delta_{L} \widetilde{q}_{-}^{n}\left(Q_{0}^{2}\right)} P_{q \bar{q}}^{(1), n}\right] /\left(\pi \beta_{0}\right)} \\
& \times\left(\frac{\alpha_{s}\left(Q^{2}\right)}{\alpha_{s}\left(Q_{0}^{2}\right)}\right)^{-2\left(\Delta_{T} P_{q q}^{(0), n}-P_{q q}^{(0), n}\right) / \beta_{0}} \frac{2\left|\Delta_{T} \widetilde{q}_{-}^{n}\left(Q_{0}^{2}\right)\right|}{\widetilde{q}_{-}^{n}\left(Q_{0}^{2}\right)+\Delta_{L} \widetilde{q}_{-}^{n}\left(Q_{0}^{2}\right)}
\end{aligned}
$$

has to be smaller than unity in the scheme we have defined. Here we have used for the evolution of $\widetilde{q}_{-}^{n}\left(Q^{2}\right)$ $+\Delta_{L} \widetilde{q}_{-}^{n}\left(Q^{2}\right) \quad$ that $\quad[16] \quad \Delta_{L} P_{q q}^{(0), n} \equiv P_{q q}^{(0), n}$ and $[24,25] \Delta_{L} C_{q}^{\mathrm{DY}, n} \equiv C_{q}^{\mathrm{DY}, n}$ as well as $[26,27,21] \Delta_{L} P_{q q, \pm}^{(1), n}$ $\equiv P_{q q, \bar{\mp}}^{(1), n}$ where $P_{q q, \pm}^{(1), n}=P_{q q}^{(1), n} \pm P_{q \bar{q}}^{(1), n}$ is defined in analogy with (17). Clearly, the right-hand side in (26) will in general only be smaller than unity if this is the case for the input densities at $Q=Q_{0}, 2\left|\Delta_{T} \widetilde{q}_{-}^{n}\left(Q_{0}^{2}\right)\right| /\left(\widetilde{q}_{-}^{n}\left(Q_{0}^{2}\right)+\Delta_{L} \widetilde{q}_{-}^{n}\left(Q_{0}^{2}\right)\right)$ $\leqslant 1$. This, of course, is impossible to prove or disprove within perturbative QCD. What one can do (and what is the purpose of this paper) is to assume validity of Soffer's inequality for the input and see whether the inequality is preserved when going to higher $Q^{2}$. From (6) we know that the exponent $-2\left(\Delta_{T} P_{q q}^{(0), n}-P_{q q}^{(0), n}\right) / \beta_{0}$ is positive, which demonstrates preservation of Soffer's inequality at the LO level as discussed in [15]. Even more, one finds that, for any reasonably small $\alpha_{s}\left(Q_{0}^{2}\right)$,

$$
\begin{aligned}
& {\left[1-\frac{\alpha_{s}\left(Q_{0}^{2}\right)-\alpha_{s}\left(Q^{2}\right)}{\pi \beta_{0}} \frac{\beta_{1}}{2 \beta_{0}}\left(\Delta_{T} P_{q q}^{(0), n}-P_{q q}^{(0), n}\right)\right]} \\
& \times\left(\frac{\alpha_{s}\left(Q^{2}\right)}{\alpha_{s}\left(Q_{0}^{2}\right)}\right)^{-2\left(\Delta_{T} P_{q q}^{(0), n}-P_{q q}^{(0), n}\right) / \beta_{0}}
\end{aligned}
$$

remains smaller than unity. It also turns out that the unpolarized $P_{q \bar{q}}^{(1), n}$ is always negative, so that the corresponding term in the denominator of Eq. (26) will always help preserve Soffer's inequality. What still is to be shown is that the remaining terms in (26) do not cause any problem if (in the "worst" case) $2\left|\Delta_{T} \widetilde{q}_{-}^{n}\left(Q_{0}^{2}\right)\right| /\left(\widetilde{q}_{-}^{n}\left(Q_{0}^{2}\right)+\Delta_{L} \widetilde{q}_{-}^{n}\left(Q_{0}^{2}\right)\right)=1$. Expanding in $\alpha_{s}$ and taking into account the above observations one immediately finds that in this case

$$
\left(P_{q q}^{(1), n}-\Delta_{T} P_{q q,-}^{(1), n}\right)-\frac{\beta_{0}}{4}\left(C_{q}^{\mathrm{DY}, n}-\Delta_{T} C_{q}^{\mathrm{DY}, n}\right)
$$

has to be positive to guarantee validity of Soffer's inequality for valence densities at NLO. Having worked out this condition in Mellin- $n$ space for convenience, we will now return to Bjorken- $x$ space. In the following we will calculate the splitting function $\Delta_{T} P_{q q,-}^{(1)}(x)$, to see whether the $x$-space counterpart of the expression in (28),

$$
\left(P_{q q}^{(1)}(x)-\Delta_{T} P_{q q,-}^{(1)}(x)\right)-\frac{\beta_{0}}{4}\left(C_{q}^{\mathrm{DY}}(x)-\Delta_{T} C_{q}^{\mathrm{DY}}(x)\right),
$$

is indeed positive.

We emphasize that our considerations in the above equations only apply to the valence densities. We will also provide the splitting function $\Delta_{T} P_{q q,+}^{(1)}$ which is needed for the NLO evolution of the combination $\Delta_{T} q_{+}$in (19). To examine, however, the validity of Soffer's inequality for the nonvalence quark densities would involve taking into account the singlet evolution of the unpolarized and longitudinally polarized parton distributions. This can only be done within a detailed numerical study, which is beyond the scope of this paper.

\section{CALCULATION OF THE NLO TRANSVERSITY SPLITTING FUNCTIONS}

Our calculation of the NLO splitting functions for the transversity distributions closely follows the ones performed in the unpolarized [18-20] and longitudinally polarized [21] cases. We only briefly outline the method and its application to the transversity case here. A thorough overview of the technique can be found in $[18,20]$. 
The method we will use is set up in Bjørken- $x$ space. It is based on the factorization properties of mass singularities in the lightlike axial gauge, specified by introducing a light-like vector $n\left(n^{2}=0\right)$ with $n \cdot A=0, A$ being the gluon field. The general strategy then consists of first expanding the squared matrix element $\Delta_{T} M$ for the scattering of two (transversely polarized) (anti)quarks into two ladders of two-particle irreducible (2PI) kernels [28]. The crucial point is that in the light-cone gauge the 2PI kernels are finite before the integrations over the sides of the ladders are performed. Collinear singularities therefore appear only when integrating over the lines connecting the rungs of the ladders [28]. This allows for systematically projecting out the singularities by introducing a projector onto (transversely polarized) physical states, $\Delta_{T} \mathcal{P}$. More precisely, $\Delta_{T} \mathcal{P}$ decouples the product $\Delta_{T}(A B)$ of two successive $2 \mathrm{PI}$ kernels by projecting onto states of definite transverse polarization of the particle connecting the kernels and by setting this particle on-shell in kernel $A$. Transverse polarization for a quark entering a kernel is obtained by using

$$
u(p, s) \bar{u}(p, s)=-\not b \gamma_{5},
$$

for its spinor $u(p, s)$. Here $p$ is the quark's momentum and $s$ its transverse spin vector satisfying $s^{2}=-1, s \cdot p=0, s \cdot n$ $=0$, where the latter equality is a consequence of using $n$ to define the longitudinal direction,

$$
n \cdot p \equiv p n \neq 0, \quad n \cdot t=p \cdot t=0,
$$

$t$ being any transverse vector. The part of $\Delta_{T} \mathcal{P}$ that is relevant for the Dirac algebra is then found to be

$$
\Delta_{T} \mathcal{P} \sim \frac{1}{4 n \cdot k} h \hbar \gamma_{5}
$$

where $k$ is the momentum of the particle emerging from the top of the kernel. As already mentioned several times, no gluonic projection operators are to be introduced in the transversity case. By means of $\Delta_{T} \mathcal{P}, \Delta_{T} M$ can be written in the factorized form

$$
\Delta_{T} M=\Delta_{T} \hat{\sigma} \otimes \Delta_{T} \Gamma_{1} \otimes \Delta_{T} \Gamma_{2},
$$

where $\Delta_{T} \hat{\sigma}$ is interpreted as the (finite) short-distance (DrellYan) subprocess cross section, whereas the factors $\Delta_{T} \Gamma$ (one for each quark) contain all (and only) mass singularities and are process-independent. The explicit expression for $\Delta_{T} \Gamma$ in terms of the 2PI kernels can be easily derived from $[18,20]$. Working in dimensional regularization $(d=4-2 \epsilon$ dimensions) in the $\overline{\mathrm{MS}}$ scheme it can be shown [18] that the residue of the $1 / \epsilon$ pole of $\Delta_{T} \Gamma$ corresponds to the evolution kernels we are looking for:

$$
\begin{aligned}
\Delta_{T} \Gamma_{i i}\left(x, \alpha_{s}, \frac{1}{\epsilon}\right)= & \delta(1-x)-\frac{1}{\epsilon}\left(\frac{\alpha_{s}}{2 \pi} \Delta_{T} P_{q q}^{(0)}(x)\right. \\
& \left.+\frac{1}{2}\left(\frac{\alpha_{s}}{2 \pi}\right)^{2} \Delta_{T} P_{i i}^{(1)}(x)+\cdots\right)+O\left(\frac{1}{\epsilon^{2}}\right),
\end{aligned}
$$

where we have restored the subscripts $\{i i\}=\{q q, \pm\},\{\Sigma \Sigma\}$ that distinguish between the various NLO quark-to-quark splitting functions.

The Feynman graphs contributing to $\Delta_{T} \Gamma_{i i}$ at NLO are obviously the same as in the unpolarized and the longitudinally polarized calculations $[18,20,21]$ and need not be repeated here. Let us instead collect a few important points of the calculation: ${ }^{2}$

As mentioned above, the calculation relies on the use of the light-cone gauge. Following $[18,20,21]$, we use the principal value prescription to regularize the spurious singularities resulting from the gauge propagator (see [20] for details).

From Eqs. (30),(32) we see that the Dirac matrix $\gamma_{5}$ enters the calculation via the projectors on physical states of transverse polarization of the quarks. In general, this can lead to complications when dimensional regularization is used. It turns out, however, that no problems related to $\gamma_{5}$ occur in our calculation due to the fact that all Dirac traces that we need contain two $\gamma_{5}$ matrices, one coming from the projector $\Delta_{T} \mathcal{P}$ in (32), the other from (30). In this case, it is safe to use a fully anticommuting $\gamma_{5}$, which effectively removes the $\gamma_{5}$ from the traces via $\gamma_{5}^{2}=1$. The only case where this does not work is the splitting function $\Delta_{T} P_{q q, P S}^{(1)}$ for which there are two separate quark lines containing one $\gamma_{5}$ each. However, it is immediately obvious that for both Dirac traces the $\gamma_{5}$ is accompanied by an odd number of other Dirac matrices. So the traces vanish irrespective of the prescription for $\gamma_{5}$. As a result, the splitting function $\Delta_{T} P_{q q, P S}^{(1)}$ vanishes identically.

The calculation is technically slightly more involved than in the unpolarized or the longitudinally polarized cases, owing to the appearance of scalar products of momenta with the transverse spin vector in the squared matrix elements. These scalar products, which are obviously always quadratic in $s$, introduce extra transverse degrees of freedom that need to be integrated out. In LO, this is a rather straightforward task. The only scalar product of this kind that occurs is $(k \cdot s)^{2}$, which after integration can be effectively replaced by

$$
(k \cdot s)^{2} \rightarrow-\frac{1}{2(1-\epsilon)} k^{2}(1-x) .
$$

This term is actually multiplied by $\epsilon$ in the LO squared matrix element. As a result, it only contributes to the LO transversity splitting function in $d=4-2 \epsilon$ dimensions, $\Delta_{T} P_{q q}^{(0), d=4-2 \epsilon}(x)$, but not to its four-dimensional counterpart. In the NLO calculation, however, the $d$-dimensional LO result $\Delta_{T} P_{q q}^{(0), d=4-2 \epsilon}(x)$ is needed. It turns out that the con-

\footnotetext{
${ }^{2}$ We use the program TRACER of [29] for calculating the Dirac traces and performing contractions.
} 
tribution from the term $\sim \boldsymbol{\epsilon}(k \cdot s)^{2}$ is cancelled by other terms $\sim \epsilon$ in the matrix element, so that for $x<1$ the $d$-dimensional LO splitting function is identical to the four-dimensional one of [2],

$$
\Delta_{T} P_{q q}^{(0), d=4-2 \epsilon}(x)=\Delta_{T} P_{q q}^{(0)}(x)=C_{F} \frac{2 x}{1-x} \quad(x<1) .
$$

Note that this result for the $d$-dimensional LO splitting function is at variance with the one of [17] in which residual terms $\sim \epsilon(1-x)$ are present, presumably resulting from the assumption $k \cdot s=0$ in [17]. We will return to this point later.

At NLO, the situation is more complicated. For the virtual graphs, besides terms involving $(k \cdot s)$ also powers of $(r \cdot s)$ appear, where $r$ is the loop momentum. To integrate these, one needs tensorial two- and three-point functions. It turns out that all the functions we need here already appeared in our unpolarized [20] and longitudinally polarized [21] calculations, so that there is no really new integral to be calculated. When calculating the squared matrix elements for the real diagrams, one encounters the terms $(k \cdot s)^{2}$, $(k \cdot s)\left(l_{1} \cdot s\right)$ and $\left(l_{1} \cdot s\right)^{2}$, where $l_{1}$ is the momentum of one of the outgoing "unobserved" particles. One can integrate these terms in two steps: first, one integrates over the terms $\left(l_{1} \cdot s\right),\left(l_{1} \cdot s\right)^{2}$. These integrations have lengthy expressions, given in detail in Appendix A. After this integration, the squared matrix element still depends on $(k \cdot s)^{2}$ for which one finds, similarly to (35):

$$
(k \cdot s)^{2} \rightarrow-\frac{1}{2(1-\epsilon)}\left(k^{2}-2 x(p \cdot k)\right) .
$$

After this substitution, the resulting expression for the matrix element squared contains just terms that are familiar from the unpolarized calculation. It can then be integrated using the techniques developed in [20,21].

For a transversely polarized antiquark with a spinor $v(p, s)$ one has expressions identical to (30),(32). Thusunlike the longitudinally polarized case [21] — there is no extra minus sign when calculating the function $\Delta_{T} P_{q \bar{q}}^{(1)}$.
The endpoint contributions to the transversity splitting functions, i.e. the contributions $\sim \delta(1-x)$, are necessarily the same as in the unpolarized and longitudinally polarized cases since they are just provided by the residue of the pole of the full quark propagator $[18,20,21]$.

We are now in a position to present the final results.

\section{RESULTS} $[2]$

For completeness, we begin with the full one-loop result

$$
\Delta_{T} P_{q q}^{(0)}(x)=C_{F}\left[\frac{2 x}{(1-x)_{+}}+\frac{3}{2} \delta(1-x)\right]
$$

where the +-prescription is defined in the usual way:

$$
\int_{0}^{1} d z f(z)(g(z))_{+} \equiv \int_{0}^{1} d z(f(z)-f(1)) g(z)
$$

To write down our final result for the $\Delta_{T} P_{q q, \pm}^{(1)}$ we introduce

$$
\begin{gathered}
\delta_{T} P_{q q}^{(0)}(x)=\frac{2 x}{(1-x)_{+}}, \\
S_{2}(x)=\int_{x / 1+x}^{1 / 1+x} \frac{d z}{z} \ln \left(\frac{1-z}{z}\right) \\
=-2 \operatorname{Li}_{2}(-x)-2 \ln x \ln (1+x)+\frac{1}{2} \ln ^{2} x-\frac{\pi^{2}}{6},
\end{gathered}
$$

where $\mathrm{Li}_{2}(x)$ is the dilogarithm. We then have [cf. Eq. (17)], in the $\overline{\mathrm{MS}}$ scheme,

$$
\Delta_{T} P_{q q, \pm}^{(1)}(x) \equiv \Delta_{T} P_{q q}^{(1)}(x) \pm \Delta_{T} P_{q q}^{(1)}(x)
$$

where ${ }^{3}$

$$
\begin{aligned}
\Delta_{T} P_{q q}^{(1)}(x)= & C_{F}^{2}\left[1-x-\left(\frac{3}{2}+2 \ln (1-x)\right) \ln x \delta_{T} P_{q q}^{(0)}(x)+\left(\frac{3}{8}-\frac{\pi^{2}}{2}+6 \zeta(3)\right) \delta(1-x)\right]+\frac{1}{2} C_{F} N_{C}[-(1-x) \\
& \left.+\left(\frac{67}{9}+\frac{11}{3} \ln x+\ln ^{2} x-\frac{\pi^{2}}{3}\right) \delta_{T} P_{q q}^{(0)}(x)+\left(\frac{17}{12}+\frac{11 \pi^{2}}{9}-6 \zeta(3)\right) \delta(1-x)\right]+\frac{2}{3} C_{F} T_{f}\left[\left(-\ln x-\frac{5}{3}\right) \delta_{T} P_{q q}^{(0)}(x)\right. \\
& \left.-\left(\frac{1}{4}+\frac{\pi^{2}}{3}\right) \delta(1-x)\right] \\
& \Delta_{T} P_{q \bar{q}}^{(1)}(x)=C_{F}\left(C_{F}-\frac{1}{2} N_{C}\right)\left[-(1-x)+2 S_{2}(x) \delta_{T} P_{q q}^{(0)}(-x)\right]
\end{aligned}
$$

\footnotetext{
${ }^{3}$ Needless to say that the + -prescription is not needed if the function multiplying the factor $1 /(1-x)_{+}$is vanishing at $x=1$. Neither is it to be taken into account in the function $\delta_{T} P_{q q}^{(0)}(-x)$.
} 
where $\quad C_{F}=4 / 3, \quad N_{C}=3, \quad T_{f}=f T_{R}=f / 2 \quad$ and $\quad \zeta(3)$ $\approx 1.202057$.

For checking Soffer's inequality via Eq. (29) we still need the $\mathcal{O}\left(\alpha_{s}\right)$ corrections to the short-distance subprocess cross section for the transversely polarized Drell-Yan, $\Delta_{T} C_{q}^{\mathrm{DY}}(x)$, in the $\overline{\mathrm{MS}}$ scheme. The corresponding result was first presented in [9] where, however, dimensional reduction rather than dimensional regularization was used. The translation of the result to dimensional regularization was provided in [17]. As a check of the expression in [17], we employ an earlier result for the transversely polarized Drell-Yan cross section at $\mathcal{O}\left(\alpha_{s}\right)$ which was obtained in [8] by assuming off-shell gluons to regularize the appearing poles. Studying in detail the structure of the collinear singularities for both dimensional and off-shell regularization, it is straightforward to transform the result from one regularization scheme to the other. Starting from the result in [8], we obtain in this way for the $\overline{\mathrm{MS}}$ scheme:

$$
\begin{aligned}
\Delta_{T} C_{q}^{\mathrm{DY}}(x)= & C_{F}\left[8 x\left(\frac{\ln (1-x)}{1-x}\right)_{+}-\frac{4 x \ln x}{1-x}-\frac{6 x \ln ^{2} x}{1-x}\right. \\
& \left.+4(1-x)+\left(\frac{2}{3} \pi^{2}-8\right) \delta(1-x)\right]
\end{aligned}
$$

This result indeed coincides with the one in [17] for the choice $\Delta_{T} d=-\delta(1-x)$ in that paper. On the other hand, the expression for $\Delta_{T} d$ that is claimed in [17] to provide the link to the $\overline{\mathrm{MS}}$ scheme (in dimensional regularization) is $\Delta_{T} d$ $=-\delta(1-x)+2(1-x)$. The reason for this difference lies in the discrepancy between our calculation and the one of [17] for the $(4-2 \epsilon)$-dimensional LO splitting function [see the remark after Eq. (36)]. We note that the correctness of our result (36) for this quantity is corroborated by the observation that the $d$-dimensional $2 \rightarrow 3$ matrix element squared for the process $\vec{q} \vec{q} \rightarrow \mu^{+} \mu^{-} g$ (with transversely polarized incoming (anti)quarks) nicely factorizes into the product of the $d$-dimensional $2 \rightarrow 2$ matrix element squared for $\vec{q} \vec{q} \rightarrow \mu^{+} \mu^{-}$times the splitting function $\Delta_{T} P_{q q}^{(0), d=4-2 \epsilon}$ of Eq. (36), when the collinear limit of the gluon to be parallel to one of the incoming quarks is taken properly. This clearly demonstrates again the correctness of (36). The result of [17] for the transversely polarized Drell-Yan cross section at NLO (obtained for dimensional regularization) therefore necessarily corresponds to a different (non- $\overline{\mathrm{MS}}$ ) factorization scheme.

The remaining NLO ingredients for Eq. (29) are the unpolarized quantities $P_{q q}^{(1)}(x)$ and $C_{q}^{\mathrm{DY}}(x)$, which can be found in $[18,20]$ and $[30]$, respectively. Inserting everything into Eq. (29) it turns out that all distributions at $x=1$ (+-prescriptions and $\delta$-functions) drop out, so that everything is regular at $x=1$. We can therefore plot the result for

$$
\widetilde{D}(x) \equiv\left(P_{q q}^{(1)}(x)-\Delta_{T} P_{q q,-}^{(1)}(x)\right)-\frac{\beta_{0}}{4}\left(C_{q}^{\mathrm{DY}}(x)-\Delta_{T} C_{q}^{\mathrm{DY}}(x)\right)
$$

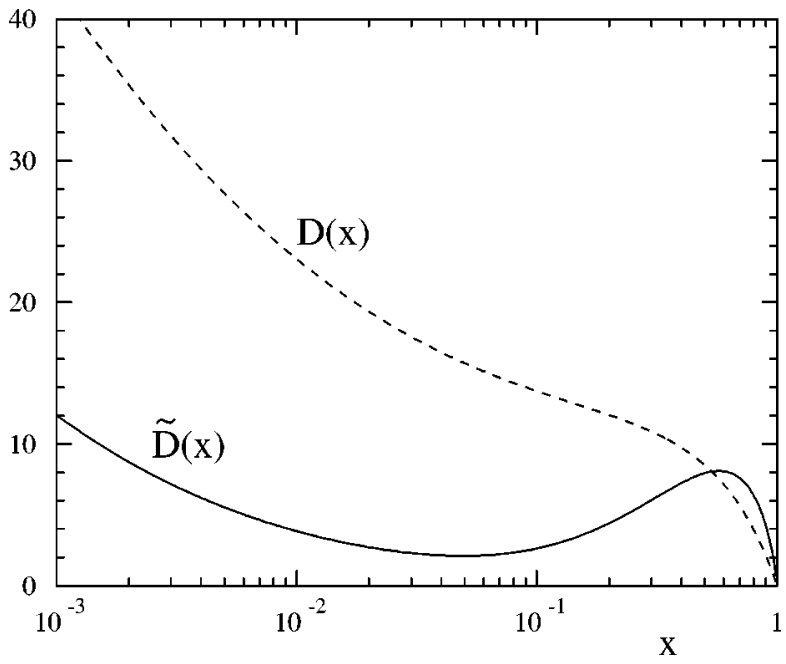

FIG. 1. The functions $\widetilde{D}(x), D(x)$ [see Eqs. (46),(49)] for $f$ $=3$ active flavors.

directly in $x$-space. This is done in Fig. 1 for $f=3$ active flavors. One can clearly see that $\widetilde{D}(x)$ is always positive, which demonstrates the validity of Soffer's inequality for valence densities at the NLO level in the sense that if Soffer's inequality is valid at the input scale, it will not be broken at any higher $Q^{2}$. As becomes visible from Fig. 1, $\widetilde{D}(x)$ diverges at $x \rightarrow 0$. This feature is a consequence of a rather peculiar small- $x$ behavior of the NLO transversity splitting functions and subprocess cross sections: the quantities in Eqs. (43),(44),(45) all have a constant limiting behavior at $x \rightarrow 0$. All terms that rise logarithmically in $x$ are dampened by a factor $x$ from the "LO", splitting term $\delta_{T} P_{q q}^{(0)}(x)$. This behavior is in contrast to the one for $P_{q q}^{(1)}(x), C_{q}^{\mathrm{DY}}(x)$ in (46), hence the rise of $\widetilde{D}(x)$ at small $x$. More precisely, we find from (43),(44) for $x \rightarrow 0$

$$
\begin{gathered}
\Delta_{T} P_{q q,-}^{(1)}(x) \approx C_{F}\left(2 C_{F}-N_{C}\right)+2 C_{F}^{2} x \ln ^{2} x, \\
\Delta_{T} P_{q q,+}^{(1)}(x) \approx-2 C_{F}\left(C_{F}-N_{C}\right) x \ln ^{2} x,
\end{gathered}
$$

that is, the constant terms even cancel in $\Delta_{T} P_{q q,+}^{(1)}(x)$. We note that the logarithmic terms in $(47),(48)$ can be recovered from Eq. (3.15) of [31] where a resummation of small- $x$ double-logarithms for the transversity densities was performed. ${ }^{4}$ The constant terms in (47), however, were not anticipated to appear in ordinary Altarelli-Parisi evolution in [31]. The very mild small- $x$ behavior of Eqs. (47),(48) will lead to a strong suppression of the transversity distributions with respect to the unpolarized and longitudinally polarized ones during $Q^{2}$ evolution at small $x$.

As discussed in Sec. II we have chosen a factorization scheme in which the NLO Drell-Yan cross sections for the unpolarized, the longitudinally polarized and the transversely

\footnotetext{
${ }^{4}$ Here one has to correct for a missing factor $1 / 2$ in front of the term $f_{V}^{-}(\omega)$ in Eq. (3.15) of [31] that got lost in the derivation of this equation from [32]. I am thankful to A. Vogt for helpful communications on this point.
} 


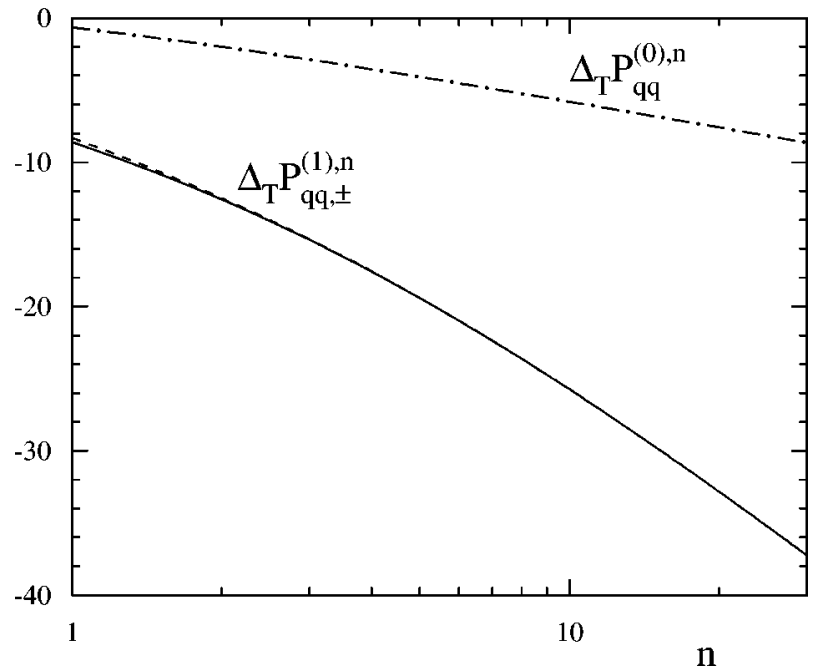

FIG. 2. The Mellin- $n$ moments of the NLO transversity splitting functions $\Delta_{T} P_{q q,-}^{(1)}$ (solid line) and $\Delta_{T} P_{q q,+}^{(1)}$ (dashed line) versus real $n \geqslant 1$. For comparison the dash-dotted curve shows the LO result $\Delta_{T} P_{q q}^{(0), n}$.

polarized cases all retain their respective LO forms. We have done this to deal with quantities that are manifestly schemeindependent. From a practical point of view, this may not be very useful since the unpolarized and longitudinally polarized parton densities are usually defined in the $\overline{\mathrm{MS}}$ scheme. In particular, when building models for the transversity densities, one would like to know whether Soffer's inequality is also preserved under NLO evolution in the $\overline{\mathrm{MS}}$ scheme. If this is the case then it appears likely to make sense to assume validity of Soffer's inequality also for the $\overline{\mathrm{MS}}$ NLO input parton densities and not only for the parton densities defined in our scheme. For this reason the dashed line in Fig. 1 shows only the quantity

$$
D(x) \equiv P_{q q}^{(1)}(x)-\Delta_{T} P_{q q,-}^{(1)}(x),
$$

which corresponds to performing the evolution in the $\overline{\mathrm{MS}}$ scheme. As can be seen, Soffer's inequality will also be maintained here.

For convenience, we present the Mellin- $n$ moments of the transversity NLO quantities $\Delta_{T} P_{q q, \pm}^{(1)}, \Delta_{T} C_{q}^{\mathrm{DY}}$ in Appendix $B$. These are useful for a numerical evaluation of our results. Figure 2 shows the moments of the splitting functions versus real $n \geqslant 1$. As can be seen, the moments are all negative. Furthermore, the numerical contribution of the splitting function $\Delta_{T} P_{q \bar{q}}^{(1), n}$ is very small for $n \geqslant 1$. For comparison we also show the moments of the LO transversity splitting function. The expression for the first $(n=1)$ moment of $\Delta_{T} P_{q q,-}^{(1)}$ turns out to be

$$
\Delta_{T} P_{q q,-}^{(1), 1}=\frac{19}{8} C_{F}^{2}-\frac{257}{72} C_{F} N_{C}+\frac{13}{18} C_{F} T_{f} .
$$

This quantity participates in the NLO evolution of the nucleon's tensor charge which is given by the first moment of the sum of the valence transversity densities [3].

\section{CONCLUSIONS}

We have presented a calculation of the next-to-leading order splitting functions $\Delta_{T} P_{q q, \pm}^{(1)}(x)$ for the $Q^{2}$ evolution of transversity parton densities. The method we have used here is the one of $[28,18]$ which was applied to the unpolarized and longitudinally polarized cases in $[18,19]$ and [21], respectively. We have used our results to examine the preservation of Soffer's inequality for valence quark densities under NLO $Q^{2}$ evolution. It turned out that (in a suitably defined factorization scheme) the inequality is indeed maintained at any higher $Q^{2}$ if it is satisfied at the input scale, which provides strong support for its correctness. In other words, $\mathcal{O}\left(\alpha_{s}\right)$ corrections do not seem to invalidate the inequality. We note again that extending our results to nonvalence quark densities affords inclusion of the full singlet evolution of the unpolarized and longitudinally polarized densities and thus has to be subject to a detailed numerical study. Combining our finding for the valence part with previous experience from $\mathrm{LO}$ [15] indicates that no surprises concerning Soffer's inequality are expected in the singlet sector.

Note added. After completing this work we received the papers $[33,34]$ in which the two-loop anomalous dimensions for the transversity distributions were calculated using the operator product expansion. The obtained results should correspond to the NLO splitting functions we have calculated. Indeed, our results in Mellin- $n$ space in Eq. (B1) are exactly identical to those in [34] and [33] (after revision of that paper).

\section{ACKNOWLEDGMENTS}

I am grateful to J. C. Collins, X. Ji, J. Soffer and M. Stratmann for helpful discussions and to B. Kamal for useful correspondence on his calculation of the NLO corrections to the transversely polarized Drell-Yan cross section.

\section{APPENDIX A}

In this appendix we provide the expressions for the integrations over the terms $\left(l_{1} \cdot s\right)$ and $\left(l_{1} \cdot s\right)^{2}$. Defining

$$
\lambda \equiv 2\left(-(1-x)\left(l_{1} \cdot p\right)+\frac{k^{2}}{2}-(p \cdot k)\left(1-\frac{l_{1} \cdot n}{p n}\right)\right),
$$

we find

$$
\left(l_{1} \cdot s\right) \rightarrow-\frac{1}{2} \frac{\lambda}{k^{2}-2 x(p \cdot k)}(k \cdot s),
$$




$$
\begin{aligned}
\left(l_{1} \cdot s\right)^{2} \rightarrow & \frac{1}{1-2 \epsilon}\left(2\left(l_{1} \cdot p\right) \frac{l_{1} \cdot n}{p n}+\frac{\lambda^{2}}{4\left(k^{2}-2 x(p \cdot k)\right)}\right) \\
& \times\left[1+\frac{(k \cdot s)^{2}}{k^{2}-2 x(p \cdot k)}\right]+\frac{\lambda^{2}(k \cdot s)^{2}}{4\left(k^{2}-2 x(p \cdot k)\right)^{2}}
\end{aligned}
$$

as the effective substitutions in the squared matrix element. For kinematics and the notation of the momenta see [21].

\section{APPENDIX B}

In this appendix we present the Mellin- $n$ moments of the NLO quantities $\Delta_{T} P_{q q, \pm}^{(1)}(x), \Delta_{T} C_{q}^{\mathrm{DY}}(x)$ (in the $\overline{\mathrm{MS}}$ scheme) which are useful for a numerical evaluation of our results:

$$
\begin{aligned}
\Delta_{T} P_{q q, \eta}^{(1), n}= & C_{F}^{2}\left[\frac{3}{8}+\frac{1-\eta}{n(n+1)}-3 S_{2}(n)-4 S_{1}(n)\left(S_{2}(n)\right.\right. \\
& \left.\left.-S_{2}^{\prime}\left(\frac{n}{2}\right)\right)-8 \widetilde{S}(n)+S_{3}^{\prime}\left(\frac{n}{2}\right)\right]+\frac{1}{2} C_{F} N_{C}\left[\frac{17}{12}\right. \\
& -\frac{1-\eta}{n(n+1)}-\frac{134}{9} S_{1}(n)+\frac{22}{3} S_{2}(n)+4 S_{1}(n) \\
& \left.\times\left(2 S_{2}(n)-S_{2}^{\prime}\left(\frac{n}{2}\right)\right)+8 \widetilde{S}(n)-S_{3}^{\prime}\left(\frac{n}{2}\right)\right]
\end{aligned}
$$

$$
\begin{aligned}
& +\frac{2}{3} C_{F} T_{f}\left[-\frac{1}{4}+\frac{10}{3} S_{1}(n)-2 S_{2}(n)\right], \\
\Delta_{T} C_{q}^{\mathrm{DY}, n}= & C_{F}\left[\frac{4}{n(n+1)}+4 S_{1}^{2}(n)+12\left(S_{3}(n)-\zeta(3)\right)-8\right. \\
& \left.+\frac{4}{3} \pi^{2}\right]
\end{aligned}
$$

where $\eta \equiv \pm$. The sums appearing here are defined by

$$
\begin{gathered}
S_{k}(n) \equiv \sum_{j=1}^{n} \frac{1}{j^{k}}, \\
S_{k}^{\prime}\left(\frac{n}{2}\right) \equiv 2^{k-1} \sum_{j=1}^{n} \frac{1+(-1)^{j}}{j^{k}} \\
\widetilde{S}(n) \equiv \sum_{j=1}^{n} \frac{(-1)^{j}}{j^{2}} S_{1}(j) .
\end{gathered}
$$

Their analytic continuations to arbitrary Mellin- $n$ (which depend on $\eta$ ) can be found in [35].
[1] J. P. Ralston and D. E. Soper, Nucl. Phys. B152, 109 (1979).

[2] X. Artru and M. Mekhfi, Z. Phys. C 45, 669 (1990).

[3] R. L. Jaffe and X. Ji, Phys. Rev. Lett. 67, 552 (1991); Nucl. Phys. B375, 527 (1992).

[4] J. L. Cortes, B. Pire, and J. P. Ralston, Z. Phys. C 55, 409 (1992).

[5] R. L. Jaffe and A. Manohar, Phys. Lett. B 223, 218 (1989); X. Ji, ibid. 289, 137 (1992).

[6] J. Soffer and O. V. Teryaev, Phys. Rev. D 56, 1353 (1997).

[7] D. Sivers, Phys. Rev. D 51, 4880 (1995).

[8] W. Vogelsang and A. Weber, Phys. Rev. D 48, 2073 (1993).

[9] A. P. Contogouris, B. Kamal, and Z. Merebashvili, Phys. Lett. B 337, 169 (1994).

[10] X. Ji, Phys. Lett. B 284, 137 (1992); R. L. Jaffe and N. Saito, ibid. 382, 165 (1996); O. Martin and A. Schäfer, Z. Phys. A 358, 429 (1997); V. Barone, T. Calarco, and A. Drago, Phys. Rev. D 56, 527 (1997).

[11] RHIC Spin Collaboration, D. Hill et al., Letter of intent RHICSPIN-LOI-1991, updated 1993; G. Bunce et al., Part. World 3, 1 (1992).

[12] J. Soffer, Phys. Rev. Lett. 74, 1292 (1995).

[13] G. R. Goldstein, R. L. Jaffe, and X. Ji, Phys. Rev. D 52, 5006 (1995).

[14] B. Kamal, A. P. Contogouris, and Z. Merebashvili, Phys. Lett. B 376, 290 (1996).

[15] V. Barone, Phys. Lett. B 409, 499 (1997).

[16] G. Altarelli and G. Parisi, Nucl. Phys. B126, 298 (1977).

[17] B. Kamal, Phys. Rev. D 53, 1142 (1996).
[18] G. Curci, W. Furmanski, and R. Petronzio, Nucl. Phys. B175, 27 (1980).

[19] W. Furmanski and R. Petronzio, Phys. Lett. 97B, 437 (1980).

[20] R. K. Ellis and W. Vogelsang, CERN-TH/96-50, RAL-TR96-012, hep-ph/9602356 (unpublished).

[21] W. Vogelsang, Phys. Rev. D 54, 2023 (1996); Nucl. Phys. B475, 47 (1996).

[22] I. Schmidt and J. Soffer, Phys. Lett. B 407, 331 (1997).

[23] M. Glück and E. Reya, Phys. Rev. D 25, 1211 (1982).

[24] P. Ratcliffe, Nucl. Phys. B223, 45 (1983).

[25] A. Weber, Nucl. Phys. B382, 63 (1992).

[26] M. Stratmann, W. Vogelsang, and A. Weber, Phys. Rev. D 53, 138 (1996).

[27] M. Glück, E. Reya, M. Stratmann, and W. Vogelsang, Phys. Rev. D 53, 4775 (1996).

[28] R. K. Ellis, H. Georgi, M. Machacek, H. D. Politzer, and G. G. Ross, Phys. Lett. 78B, 281 (1978); Nucl. Phys. B152, 285 (1979).

[29] M. Jamin and M. E. Lautenbacher, Comput. Phys. Commun. 74, 265 (1993).

[30] G. Altarelli, R. K. Ellis, and G. Martinelli, Nucl. Phys. B157, 461 (1979).

[31] R. Kirschner, L. Mankiewicz, A. Schäfer, and L. Szymanowski, Z. Phys. C 74, 501 (1997).

[32] R. Kirschner and L. N. Lipatov, Nucl. Phys. B213, 122 (1983).

[33] S. Kumano and M. Miyama, Phys. Rev. D 56, 2504 (1997).

[34] A. Hayashigaki, Y. Kanazawa, and Y. Koike, Phys. Rev. D 56, 7350 (1997).

[35] M. Glück, E. Reya, and A. Vogt, Z. Phys. C 48, 471 (1990). 